\title{
Quantitative expression analysis of selected transcription factors in pavement, basal and trichome cells of mature leaves from Arabidopsis thaliana
}

\author{
Martin Schliep • Berit Ebert • Ulrike Simon-Rosin • \\ Daniela Zoeller • Joachim Fisahn
}

Received: 12 November 2009 / Accepted: 15 December 2009/Published online: 26 January 2010

(C) The Author(s) 2009. This article is published with open access at Springerlink.com

\begin{abstract}
Gene expression levels of several transcription factors from Arabidopsis thaliana that were described previously to be involved in leaf development and trichome formation were analysed in trichome, basal and pavement cells of mature leaves. Single cell samples of these three cells types were collected by glass micro-capillaries. Realtime reverse transcription (RT)-PCR was used to analyse expression patterns of the following transcription factors: MYB23, MYB55, AtHB1, FILAMENTOUS FLOWER (FIL)/YABBY1 (YAB1), TRIPTYCHON (TRY) and CAPRICE (CPC). A difference in the expression patterns of TRY and CPC was revealed. Contrary to the CPC expression pattern, no transcripts of TRY could be detected in pavement cells. FIL/YAB1 was exclusively expressed in trichome cells. AtHB1 was highly expressed throughout all three cell types. MYB55 was higher expressed in basal cells than in trichome and pavement cells. MYB23 showed a pattern of low expression in pavement cells, medium in basal cells and high expression in trichomes. Expression patterns obtained by single cell sampling and real-time RTPCR were compared to promoter GUS fusions of the selected transcription factors. Therefore, we regenerated two transgenic Arabidopsis lines that expressed the GUS reporter gene under control of the promoters of MYB55 and YAB1. In conclusion, despite their function in leaf morphogenesis, all six transcription factors were detected in mature leaves. Furthermore, single cell sampling and promoter GUS staining patterns demonstrated the predom-
\end{abstract}

\footnotetext{
M. Schliep • B. Ebert • U. Simon-Rosin · D. Zoeller •

J. Fisahn $(\bowtie)$

Max-Planck-Institut fuer Molekulare Pflanzenphysiologie,

Am Muehlenberg 1,

14476 Potsdam, Germany

e-mail: fisahn@mpimp-golm.mpg.de
}

inant presence of MYB55 in basal cells as compared to pavement cells and trichomes.

Keywords Arabidopsis thaliana $\cdot$ Real-time RT-PCR .

Single cell $\cdot$ Transcription factors $\cdot$ Epidermis

\section{Introduction}

Quantitative information about the solute content in individual cells became accessible by application of the cell-pressure probe (Hüsken et al. 1978) that evolved from an instrument for measuring cell-water relations parameters into a micro-sampling device. A set of techniques collectively known as single cell sampling and analysis (SiCSA; Tomos and Leigh 1999) was developed and provides the basis for this application. Early extensive application of SiCSA had been to barley leaves. In particular, it demonstrated differences in metabolite composition between epidermal, mesophyll and bundle sheath cells (Fricke et al. 1994). This approach was complemented by correlating cell-specific metabolite composition with the expression pattern of genes involved in sucrose metabolism (Gallagher et al. 2001; Koroleva et al. 2001). Furthermore, Laval et al. (2002) pioneered the comparison of micro-capillary derived single cell expression data with results of promoter reporter expression studies.

To investigate the potential of single cell micro-capillary sampling in combination with quantitative reverse transcription (qRT)-PCR for transcription factor profiling of individual epidermal cells, we selected six transcription factors that are involved in leaf morphogenesis: MYB23, MYB55, TRY, CPC, AtHB1 and Fil/YAB. Myb23 is a member of the R2R3 MYB family that is involved in many different biological processes (Stracke et al. 2001), includ- 
ing trichome and root-hair differentiation, cell-shape determination, regulation of leaf form, control of secondary metabolism, pathogen response, drought stress response, protection from UV radiation and hormone signalling. Presented data indicate that the diversification of GL1 and MYB23 gene functions occurred at the level of cisregulatory sequences with respect to trichome initiation and that, in parallel, the diversification with respect to regulation of trichome branching also involved changes in respective proteins (Tominaga et al. 2007). In contrast to MYB23, MYB55 has not been characterised up to now.

CPC, as well as TRY, encodes single R3-repeat MYBlike transcription factors (TF) lacking the transcriptional activation domain (Wada et al. 1997). CPC is well characterised as being an important regulator in root-hair development, which shows striking similarities to trichome cell fate determination. Because mutations in TRY result in a few clustered leaf trichomes, TRY has been proposed to play a role in the putative inhibition pathway of trichome development (Szymanski et al. 2000).

YABBY genes constitute a small family of transcription factors with six members in Arabidopsis (Toriba et al. 2007). YABBY genes exhibit two conserved domains: a zinc-finger domain in the N-terminal region and a YABBY domain (helix-loop-helix motif) in the C-terminal region. It has been demonstrated that the FIL protein has activity to bind DNA and that YABBY proteins are localised in the nucleus (Jang et al. 2004). For rice, it has been suggested that the function of OsYABBY1 is involved in regulating the differentiation of a few specific cell types and is unrelated to regulation of axis orientation in lateral organ development (Bowman and Smyth 1999).

Homeo domain Leu zipper (HDZip) proteins represent a large family of transcription factors (Henriksson et al. 2005). These contain a DNA-binding homeo domain (HD) and an adjacent Leu zipper (Zip) motif, which mediates protein-dimer formation. HDZip proteins are apparently unique to plants but related to $\mathrm{HD}$ proteins of other eukaryotes. ATHB1 is a member of the HDZIP I gene family of Arabidopsis. These genes are less characterised with regard to their function. Present information on HDZip I and II genes suggests that some of the genes are participating in communication between environmental conditions and regulation of plant growth and development. Phenotypic analyses of plants with increased expression levels of ATHB1, $-3,-13,-20$ or -23 (HDZip I) indicate that these genes are involved in the regulation of cotyledon and leaf development (Aoyama et al. 1995).

Leaf epidermal layers of Arabidopsis thaliana consist of several highly specialised cell types, i.e. pavement cells, basal cells, trichomes and stomata (Glover 2000). Three of these cell types have been partially investigated by single cell sampling technologies. As expected, transcripts encod- ing proteins specific to individual cell types could be identified by nylon array filters and Affymetrix chips (Brandt et al. 2002). However, TFs can act in very low concentrations. As a consequence, expression data derived from high throughput methods often failed to provide information on transcription factor activity. Thus, more accurate methods are needed for the detection of low abundant transcripts that can operate in a cell autonomous manner. Solutions to this methodical drawback emerged from recent developments in real-time RT-PCR that exhibit outstanding sensitivity in TF identification. Czechowski et al. (2004) demonstrated that detection by RT-PCR can be up to 100 -fold more sensitive than micro-array experiments (Horak and Snyder 2002). To explore the power of realtime RT-PCR at the single cell level, we collected cell contents of single pavement, basal and trichome cells of fully grown leaves from $A$. thaliana and studied the expression patterns of six selected TFs by real-time RT-PCR.

\section{Materials and methods}

\section{Plant material}

A. thaliana (Col-0) plants were propagated in a greenhouse at $60 \%$ humidity undergoing a photoperiod of $16 \mathrm{~h}$ light $\left(200 \mu \mathrm{mol} \mathrm{m} \mathrm{m}^{-2} \mathrm{~s}^{-1}, 21^{\circ} \mathrm{C}\right)$ and $8 \mathrm{~h}$ darkness $\left(17^{\circ} \mathrm{C}\right)$. Plants were grown in individual pots on soil (Einheitserde $\mathrm{T}$, Tandau, Wansdorf, Germany) and watered daily. Cells were sampled from mature rosette leaves after 6 weeks.

\section{Single cell sampling}

For collecting single cell sap from leaves of $A$. thaliana sampling was performed as described previously (Brandt et al. 1999; Ebert et al. 2008; Kryvych et al. 2008). Borosilicate glass capillaries (WPI, 1B100-3: Berlin, Germany) were pulled on a List pipette puller (Darmstadt, Germany) with a tip aperture of $1-10 \mu \mathrm{m}$. Capillaries were mounted on a micro-manipulator, and leaves of an intact plant were fixed under an Optiphot 2 microscope (Nikon, Duesseldorf, Germany). A micro-capillary was inserted into a single cell via an Eppendorf remote controlled micromanipulator for sample collection (Hamburg, Germany). Immediately after withdrawal of the capillary, its contents were released into a sterile $0.5-\mathrm{ml}$ reaction vial containing 1 $\mu 1$ DEPC water with $5 \mathrm{U}$ RNase inhibitor (RNAsin, Promega, Heidelberg, Germany). Each capillary was used for only one sampling process. Extracts from five individual cells were collected into the same vial. To obtain sufficient biological replicates, we sampled 30 individual cells to produce six biological independent reproductions. 


\section{Real-time RT-PCR}

cDNA synthesis was realised on cell sap from five selected single cells. Reverse transcription reactions were performed with SuperScript ${ }^{\mathrm{TM}}$ II reverse transcriptase (Invitrogen, Karlsruhe, Germany) according to the manufacturer's instructions. Primers were designed using the program Primer Express ${ }^{\mathrm{TM}}$ (Applied Biosystems, Forster City, CA, USA). The elongation factor EF- $1 \alpha$ was used as housekeeping gene as expression reference (Nicot et al. 2005). EF- $1 \alpha$ exhibited the most constant expression level among five house-keeping genes that were tested. Realtime RT-PCR reactions were prepared according to the following protocol: $12.5 \mu 12 \times$ SYBR $^{\mathrm{TM}}$ Green PCR Mix (Applied Biosystems), $500 \mathrm{nM}$ forward primer, $500 \mathrm{nM}$ reverse primer, cDNA template from five sampled cells and $25 \mu 1 \mathrm{H}_{2} \mathrm{O}$. A master mix of sufficient cDNA and 2 SYBR ${ }^{\circledR}$ Green reagent was prepared prior and was dispensed into individual wells reducing pipetting errors and to ensure that each reaction contained an equal amount of cDNA. The SDS 5700 sequence detection system (Applied Biosystems) was used for the quantification of mRNA transcripts using real-time RT-PCR. The PCR protocol starts with a denaturing step for $10 \mathrm{~min}$ at $95^{\circ} \mathrm{C}$ followed by 40 cycles of $15 \mathrm{~s}$ at $95^{\circ} \mathrm{C}$ and a primer extension reaction at $60^{\circ} \mathrm{C}$ for $1 \mathrm{~min}$. After the run, the entire mix was denatured for $5 \mathrm{~min}$ at $95^{\circ} \mathrm{C}$ before it was slowly cooled down to $60^{\circ} \mathrm{C}$ while the kinetics of the reassociation of the complementary DNA strands of the PCR products were monitored. Re-association kinetics provided information about additional unspecific product accumulation in a dissociation curve. All PCR reactions and the negative controls were run in duplicates with three biological replicates each.

The amount of target which was normalised to the housekeeping gene was calculated according to manufacturer's protocol (Applied Biosystem) by:

$$
\begin{aligned}
\Delta \mathrm{Ct}(\text { cell type } x)= & C_{\mathrm{t}}(\text { transcript of interest }) \\
& -C_{\mathrm{t}}(\text { housekeeping gene }) .
\end{aligned}
$$

The $C_{\mathrm{t}}$ value (threshold cycle) is the PCR cycle number at which SYBR Green fluorescence in each PCR vial reaches an indiscriminate value during the exponential phase of DNA amplification (set at 0.1 in our experiments).

The following primer pairs were used:

EF-1 $\alpha \quad$ [At5g60390 (5'-TGAGCACGCTCTT CTTGCTTTCA-3' and 5'-GGTGGTGGCATCCAT CTTGTTACA-3')]; 75-bp amplicon CPC [At2g46410 (5'-TTGGCGACAGGTGGGAGTT GAT-3' and 5'-GCAAAAACGACGCCGTGTTT CATA-3')]; 97-bp amplicon
AtHB1 [At3g01470 (5'-TCCGAGGTTACTTCCCT GACCGAA-3' and 5'-GGCACTTGACCAGGTG GTTCATTA-3')]; 74-bp amplicon MYB23 [At5g40330 (5'-CACAAGCTCCTCGGCAA CAGAT- $3^{\prime}$ and 5'-CGGCTTTGACGGCAGTTGAAT GAT-3')]; 139-bp amplicon MYB55 [At4g01680 (5'-TGCCGTTCTTGGCAATA GATGGTC-3' and 5'-AAGAGCTTGTGTGTAACC GGGTC-3')]; 141-bp amplicon

TRY [At5g53200 (5'-CCATGACTCTGAAGAAGT GAGC-3' and 5'-CCCACCTATCACCGACAAGT3')]; 107-bp amplicon

FIL/YAB1 [At2g45190 (5'-TGGTACAGCAACCA CATCGGACAG-3' and 5'-GCCAAACCATCCTTG CGGTTAATG-3')]; 78-bp amplicon

All sequences were taken out of the A. thaliana transcription factor library compiled in the Max Planck Institute of Molecular Plant Physiology (Golm, Germany; Czechowski et al. 2004). For maximum specificity and efficiency, a stringent set of criteria for primer design was used. All predicted melting temperatures are $60 \pm 2^{\circ} \mathrm{C}$, the primer length was 20-24 nucleotides, the GC contents lay between $45 \%$ and $55 \%$, and the length of all amplicons was between 70 and 140 bp to obtain optimal and comparable PCR results. When it was possible, each primer pair includes one primer, spanning an intron/ exon border to ensure only cDNA amplification during PCR. Furthermore, single fragment amplification of the expected size was also controlled through 3\% agarose (SeaKem LE Agarose, Cambrex Inc., Rockland, USA) gel electrophoresis.

Construction of promoter- $\beta$-glucuronidase fusions

Genomic DNA from whole plant material was extracted using CTAB protocol (Doyle and Doyle 1987). The promoter regions of MYB55 (1.945 bp) and FIL/YAB1 $(1.752 \mathrm{bp})$ upstream of the translational start codon were amplified by PCR from genomic DNA by use of following primers:

MYB55, 5'-CCCAAGCTTGGGTTTTATCGAAG CCGTCTCCA-3'(fwd); 5'-CGGGATCCCGCTATA TATATATGTAAATAAAATACTA-3' (rev)

FIL/YAB1， 5'-GCTCTAGAGCGAGGCGACGCT TAATCACTC-3'(fwd); 5'-GCTCTAGAGCCTTTT TTGTAAGAAGGGGAAAAA-3' (rev).

The promoter sequences were inserted into the pBI101.3 plant transformation vector (Jefferson et al. 1987) using the HindIII/BamHI sites (Myb55), the XbaI/BamHI sites (AtHB1) and the $X b a \mathrm{I}$ site (FIL/YAB1) upstream of the GUS reporter gene. 


\section{A. thaliana transformation and histochemical analysis}

A. thaliana (Ler ecotype) plants were transformed by floral dip method (Clough and Bent 1998). Transformants were selected by their ability to grow on the medium containing hygromycin. Histochemical assays for GUS activity of plant tissues and organs were conducted as described previously (Jefferson et al. 1987). Histochemical GUS staining was performed on the $\mathrm{T} 2$ plants. Ten independent A. thaliana transformants were assessed for each construct.

\section{Results}

To identify cell type-specific expression levels of the transcription factors, MYB23, MYB55, AtHB1, (FIL)/ YABBY1, TRY and CPC, we collected the contents of single pavement, basal and trichome cells of $A$. thaliana leaves by glass micro-capillaries (Lieckfeldt et al. 2008; Figs. 1, 2). Subsequently, cDNA synthesis was performed on cell sap derived from pools of five individual cells. Realtime RT-PCR served to amplify and quantify the expression levels of our selected candidate genes. Gel electrophoresis of the RT-PCR products was performed for each primer set and templates derived from trichome cell samples (Fig. 3). Non-quantitative inspection of these gels indicated that MYB23 and ATH1 exhibited higher expression levels as YAB in trichomes.

Results of our quantitative RT-PCR analysis for the MYB23 transcription factor exhibited large differences in

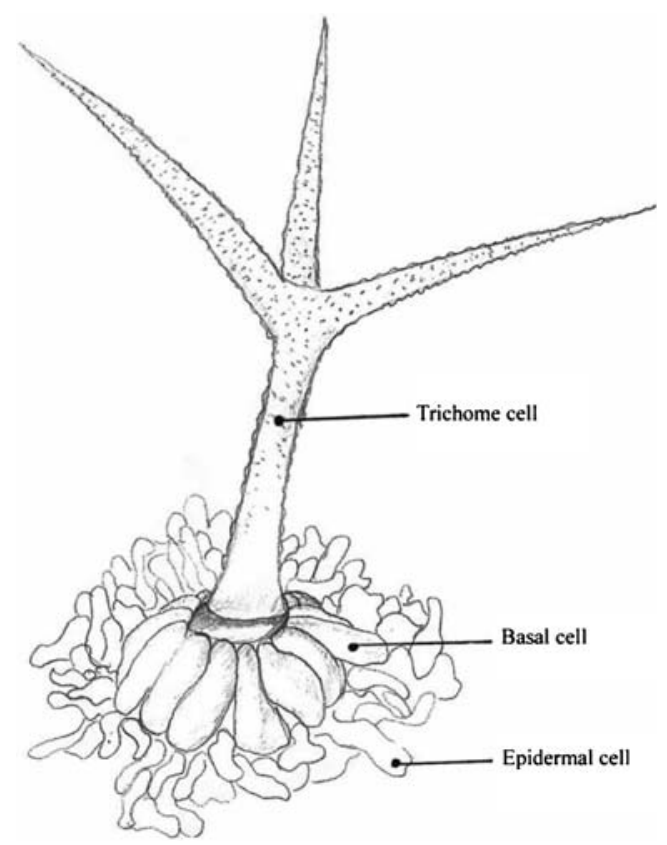

Fig. 1 Schematic drawing of a trichome cell from A. thaliana with surrounding cell files: basal and pavement cells

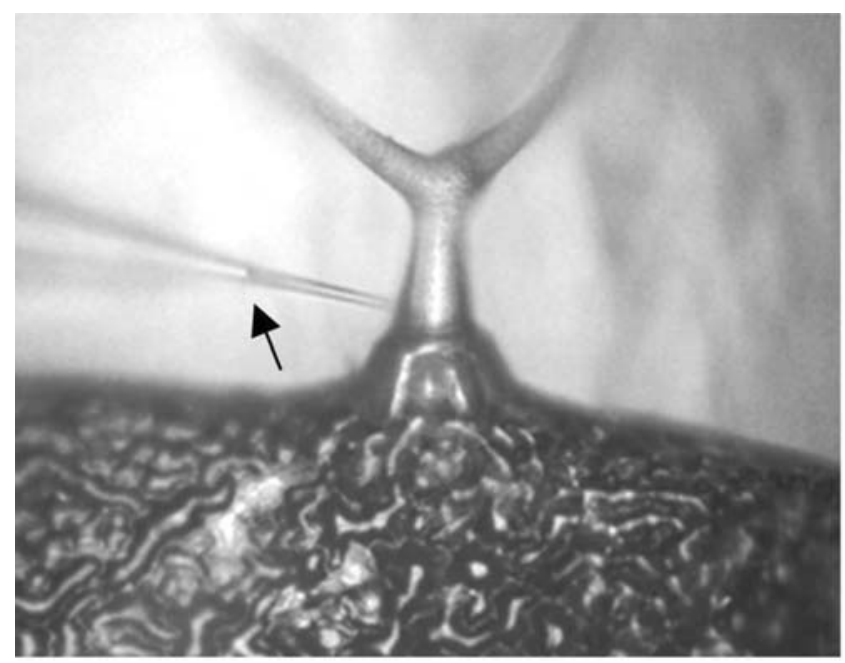

Fig. 2 A trichome from A. thaliana during cell sap sampling. A glass micro-capillary is visible on the left. The trichome is punctured, and cell sap is driven into the capillary by plant cells' turgor and capillary forces. The black arrow indicates the meniscus of cell sap in the capillary. Scale bar $50 \mu \mathrm{m}$

the three cell types (Fig. 4; Table 1). MYB23 is strongly expressed in trichomes (Fig. 4), whereas it is expressed in basal cells to a lower extent, and finally, the lowest expression of this TF occurred in pavement cells. MYB55 mRNA is stronger when expressed in basal cells than in pavement and trichome cells, which show a similar expression pattern (Fig. 4; Table 1). Since activity of the MYB55 promoter has not been assayed by promoter reporter gene fusions, we regenerated transgenic Arabidopsis lines. These lines expressed the GUS reporter gene under control of a 1.945-bp fragment upstream of the translational start codon of the MYB55 coding region. GUS activity staining was detected in pavement, basal cells and trichomes (Fig. 5). Staining intensity was most pronounced in basal cells when compared to trichomes and pavement cells (Fig. 5).

For the transcription factor FIL/YAB1, we found low expression levels in trichomes but no expression at all was detectable in basal and pavement cells (Figs. 3, 4; Table 1). To compare the expression pattern observed by microcapillary sampling, we regenerated transgenic Arabidopsis plants. These genetically modified lines expressed the GUS reporter gene under the control of a 1.752-bp promoter fragment upstream of the translational start codon of FIL/

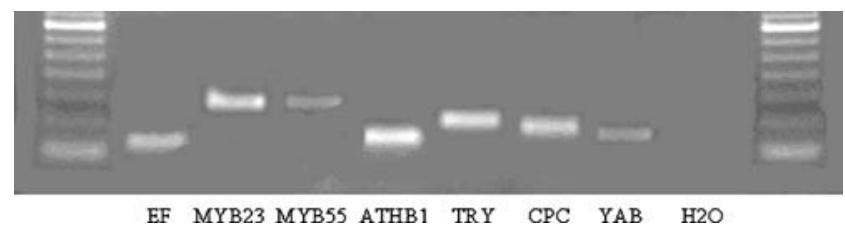

Fig. 3 Gel electrophoresis pattern of the RT-PCR products for each primer set. Templates were derived from trichome cell samples 


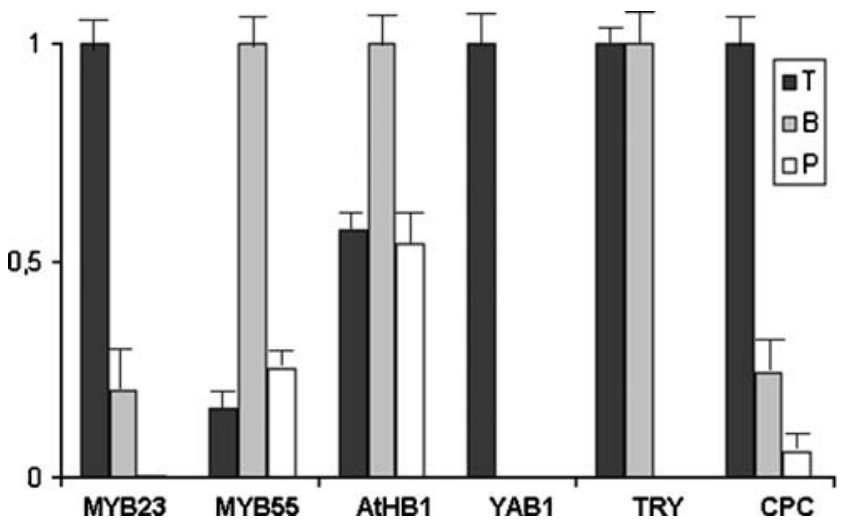

Fig. 4 Relative expression levels of transcription factors in trichome $(T)$, basal $(B)$, and pavement cells $(P)$. Highest expression of a transcription factor in either cell type was set to one and subsequently used to calculate the relative changes in the other two cell types. No bar means no transcripts could be detected in the corresponding cell type. Error bars indicate standard deviation $(n=6)$

YAB1. GUS activity staining confirmed the results of micro-capillary sampling and detection. No GUS staining was observed in pavement and basal cells. Trichomes exhibited very low GUS activity. However, the vascular bundles were marked by intensive blue colouring (Fig. 6). Overall, two staining patterns emerged with equal probability. Concerning the first category, GUS activity was absent in the base of the leaves (Fig. 6a). In the second group also, the vasculature of the leaf base was labelled by blue colour (Fig. 6b).As detected by real-time RT-PCR, AtHB1 exhibited similar mRNA expression levels in pavement, basal and trichome cells (Figs. 3, 4; Table 1).

Real-time RT-PCR of single cell extracts demonstrated that the MYB-like domain transcription factor TRY was moderately expressed in basal and trichome cells (Fig. 4; Table 1). However, we could not detect TRY mRNA in pavement cells. Expression of the transcription factor CPC was found in all three examined cell types (Fig. 4; Table 1).

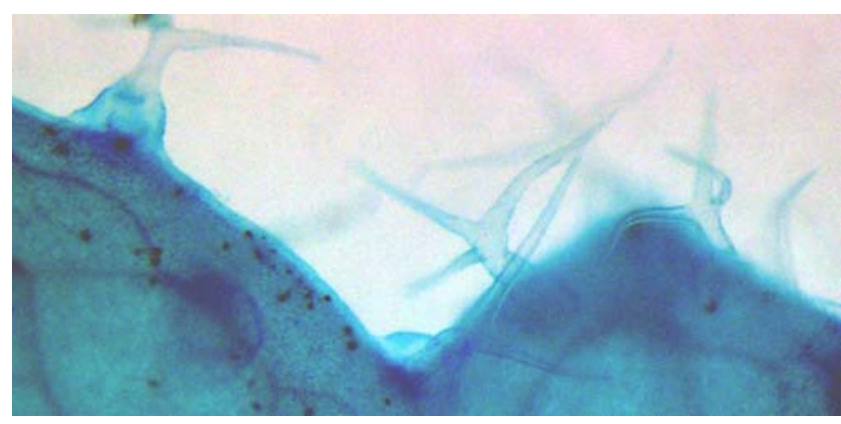

Fig. 5 Blue GUS activity staining observed in trichomes, basal and pavement cells induced by expression of the MYB55 promoter fused to the GUS reporter gene

The level of expression was higher in trichomes than in basal cells, and in both of these cell types, CPC is expressed significantly higher than in pavement cells.

\section{Discussion}

In the present study, we compared expression patterns of four MYB type, one homeo domain containing and one YABBY family member transcription factors. Expression levels of these genes were investigated in extracts collected from trichome, basal and pavement cells of $A$. thaliana (Figs. 1, 2). Due to single cell real-time RT-PCR, we succeeded in the quantification of single cell-derived transcription factor concentrations (Fig. 3; Table 1). In particular, expression levels of transcription factors can be very low and thus not be detectable by high throughput methods (Czechowski et al. 2004). Moreover, samples derived from single cells exhibit extremely small volumes in the picolitre range. Therefore, a high number of PCR cycles were needed to detect these minute amounts of transcripts within the small sample volumes (Table 1).

Table 1 Transcription factor expression levels obtained by real-time RT-PCR

\begin{tabular}{|c|c|c|c|c|c|c|c|c|c|c|c|c|}
\hline & & \multicolumn{4}{|c|}{ Trichome cells } & \multicolumn{4}{|c|}{ Basal cells } & \multicolumn{3}{|c|}{ Pavement cells } \\
\hline & & $\Delta \mathrm{Ct}$ & StdDev & $p(t / b)$ & & $\Delta \mathrm{Ct}$ & StdDev & $p(b / p)$ & & $\Delta \mathrm{Ct}$ & StdDev & $p(p / t)$ \\
\hline MYB23 & $n(6 / 6)$ & 2.94 & 0.83 & 0.008 & $n(4 / 6)$ & 5.05 & 1.05 & $8 \mathrm{E}-06$ & $n(5 / 6)$ & 11.18 & 0.51 & $1.3 \mathrm{E}-08$ \\
\hline MYB55 & $n(5 / 6)$ & 8.58 & 1.23 & 0.005 & $n(5 / 6)$ & 6.03 & 0.85 & 0.010 & $n(6 / 6)$ & 7.99 & 1.09 & 0.422 \\
\hline HAT5 & $n(6 / 6)$ & 3.61 & 0.35 & 0.172 & $n(6 / 6)$ & 2.76 & 1.37 & 0.157 & $n(6 / 6)$ & 3.74 & 0.77 & 0.712 \\
\hline YAB1 & $n(6 / 6)$ & 9.91 & 0.68 & - & n.d. & - & - & - & n.d. & - & - & - \\
\hline TRY & $n(6 / 6)$ & 4.39 & 0.67 & 0.995 & $n(5 / 6)$ & 4.38 & 1.66 & - & n.d. & - & - & - \\
\hline $\mathrm{CPC}$ & $n(6 / 6)$ & 3.55 & 0.74 & 0.001 & $n(6 / 6)$ & 5.64 & 0.83 & 0.011 & $n(6 / 6)$ & 7.47 & 1.19 & $4.4 \mathrm{E}-05$ \\
\hline
\end{tabular}

Results of single cell-specific transcription factor analysis by real-time RT-PCR. Each line represents data for one selected transcription factor. $\Delta \mathrm{Ct}$ columns show values of mean $\Delta \mathrm{Cts}$ in each of the three cell types. $p$ columns define the calculated confidence interval; in brackets, abbreviations of the two cell types compared by a two-sided $t$ test ( $t$ trichomes, $b$ basal cells, $p$ pavement cells)

$n(4 / 6)$ four out of six possible repeats realised, StdDev standard deviation of the mean $\Delta \mathrm{Ct}$, n.d. no transcripts were detected in that cell type 

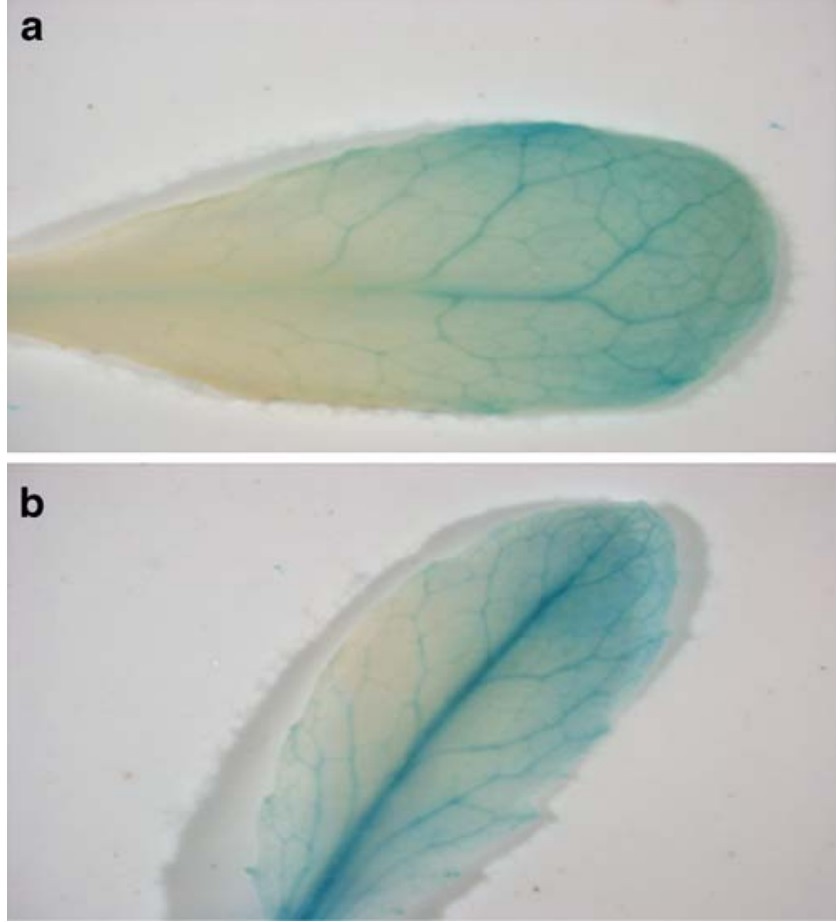

Fig. 6 a GUS activity staining in the vasculature of the leaf tip induced by expression of a YAB1 promoter fragment fused to the GUS reporter gene. b GUS staining induced by expression of the same construct observed in the vasculature of the entire leaf

MYB23 shows a high-sequence homology to GL1 and WEREWOLF (WER), which play a major role in trichome formation/root-hair growth, respectively (Tominaga et al. 2007). Since gll mutant plants lack trichomes, GL1 is thought to be a key factor in the formation of a trichome initiation activation complex. MYB23 was selected because of its partially overlapping function with GL1. However, our sampling was performed on leaf material where trichome initiation had ceased already. Therefore, we suggest that MYB23 is involved in additional processes controlling trichome function and performance. In agreement with our results obtained by gel electrophoresis (Fig. 3) and qRT-PCR (Figs. 3, 4; Table 1), strong GUS activity staining had been described in trichomes of transgenic lines expressing a MYB23 promoter GUS fusion protein (Kirik et al. 2005).

The MYB55 transcription factor is stronger expressed in basal cells than in pavement and trichome cells, which show a similar expression pattern (Fig. 4; Table 1). Up to now, there is no functional information available for this MYB-class transcription factor. Since its expression in basal cells is significantly higher than in the other two cell types, a basal cell-specific function of MYB55 might be conceivable. GUS activity staining confirmed the results our micro-sampling approach. The highest level of GUS staining emerged from basal cells in comparison to trichomes and pavement cells (Fig. 5). Thus, increased MYB55 promoter activity was demonstrated by two independent methods. Therefore, we conclude that the transcription factor MYB55 plays a predominant role in basal cells.

Expression of FIL/YAB1 in trichomes is low as indicated by real-time RT-PCR and GUS staining. Functionally, members of the YABBI family have been described to be involved in polarity specification of the adaxial/abaxial axis during leaf development (Siegfried et al. 1999). However, participation of FIL/YAB1 in cell polarity specification could link it to an early stage of trichome development. The incipient trichome cell starts to bulge out of the epidermal plane due to an inner-cellular switch from mitotic cycles to endoreduplication. Bulging of the cell leads to the rise of the trichome stalk out of the epidermis. This process requires an intra-cellular restructuring of cell polarity. For rice it has been suggested that the function of OsYABBY1 is involved in regulating the differentiation of a few specific cell types and is unrelated to polar regulation of lateral organ development (Bowman and Smyth 1999; Sawa et al. 1999). Our results obtained from promoter GUS studies are in support of the findings reported for rice. GUS activity staining was preferentially associated with the vasculature of the leaf.

TRY acts as a negative controller of trichome initiation. Try mutants exhibit $5-10 \%$ of their trichomes in clusters (up to 20 trichomes), suggesting that TRY is involved in lateral inhibition of trichome cell fate in cells surrounding an incipient trichome cell. The transcription factors GL1, TTG1 and GL3 physically interact with each other to form an activating complex (Payne et al. 2000). It is thought that TRY competes with GL1 for the same binding site to GL3, hindering the formation of sufficient activator complexes to reach the threshold level (Esch et al. 2003). The present working hypothesis for the lateral inhibition process is that the TRY gene product is somehow transported into nearby cells possibly via plasmodesmata. They are acting as a negative regulator by competing with GL1 for the same binding site to GL3. Since the phenotypical effect of trichome clusters in try mutant plants occurs in three to five cell files only directly surrounding an emerging trichome, the TRY protein is presumed to have a shortrange trichome inhibition function. This hypothesis is substantiated by our discovery that no TRY mRNA is detectable in pavement cells. The moderate expression levels of TRY mRNA in basal and trichome cells also fit the hypothesis of a post-translational, short-range inhibitor transport. Consequently, the TRY protein could be transported from TRY expressing cells to directly surrounding cell files, repressing trichome initiation there. Mature leaves of transgenic plants carrying a GUS fusion construct with the 1.4-kb TRY upstream region exhibited strong staining in 
trichomes (Schellman et al. 2002). In agreement with our qRT-PCR results, GUS staining was absent in pavement cells (Schellman et al. 2002).

CPC is well characterised as being an important regulator in root-hair development, which shows striking similarities to trichome cell fate determination. In addition, CPC and also TRY are playing a role in trichome initiation processes. Plants over-expressing CPC lack trichomes, whereas cpc mutants display an increased number of trichomes (Wada 2002). These genetic results point out that $\mathrm{CPC}$ acts as a negative regulator of trichome development. Moreover, CPC and TRY reveal a high degree of sequence homology, indicating a quite similar regulatory function. In particular, the mutant phenotypes exhibit trichome clusters in try mutants, whereas an increase in trichome number is evident in cpc mutants. It seems that the negative regulator TRY acts more area specific in a short-range around incipient trichome cells $(\sim 5$ cell files), while CPC acts on a long distance throughout the whole leaf. Comparisons of the expression patterns of CPC and TRY revealed the interesting fact that CPC is expressed in pavement cells, whereas TRY is not. This might help to explain the differences in the cpc/try mutant phenotypes, which are assumed to be due to the differing inhibitory ranges of TRY and CPC proteins. The long-range inhibition mediated by the CPC gene product could be supported by a constant level of CPC expression in pavement cells. Additionally, this finding supports the hypothesis that the TRY gene product is thought to act only in short-range inhibition. It is not expressed in pavement cells and therefore would need to be transported by some means to distant cells of an incipient trichome. Mature leaves of plants containing an expression construct where the CPC 5' regulatory sequence was fused to the GUS coding region exhibited staining in trichomes (Schellman et al. 2002). Present models of trichome differentiation and initiation in Arabidopsis leaves do not account for basal cell development. Therefore, differential expression of TRY and CPC in basal and pavement cells could provide an initial clue to the different morphologies of these two cell types. Joint presence of TRY and CPC would thus induce differentiation towards basal cell fate, whereas absence of TRY would promote pavement cell development. This suggestion would be consistent with a short-range inhibitory effect of TRY.

AtHB1 exhibited in all three cell types almost equal expression levels (Fig. 4, Table 1). These high expression levels could indicate that AtHB1 has an important function in each of the three cell types, which would also reflect its necessity to play a key role in leaf development. In transgenic tobacco plants, the expression of AtHB-1 affected the development of palisade parenchyma resulting in light green sectors in leaves and cotyledons (Aoyama et al. 1995). Palisade parenchyma normally found in wildtype leaves was absent in the light green sectors. Instead this part was occupied by cells similar in shape to the spongy mesophyll. Beyond this involvement of AtHB1 in developmental processes, our study indicates continued expression in fully differentiated epidermal cells.

\section{Conclusion}

Based on the results presented here, single cell sampling with glass micro-capillaries in combination with real-time RT-PCR provides a powerful tool to obtain single cell transcription factor expression levels in a fast and accurate way. To our knowledge, no alternative method exists at present that provides quantification of mRNA levels from transcription factors within single cells. This high degree of spatial resolution of transcript levels is, however, essential if processes involved in cell differentiation are to be understood completely. Future applications of this strategy will increase the knowledge of TF-dependent regulatory processes and how these regulators interact with each other on the single cell level. Furthermore, we demonstrated that transcription factors described to be involved in developmental processes are still expressed in the mature stages of the individual cells or organs. In particular, predominant expression of the transcription factor MYB55 in basal cells was detected by two independent methods. Moreover, TRY and CPC exhibited differential expression between basal and pavement cells.

Acknowledgements We thank Wolf Scheible, Max Planck InstituteMolecular Plant Science, Potsdam, for kindly providing the primer sequences and aliquots. Thanks to Thomas Czechowski, MPI-MP, for excellent introduction into the real-time facilities in our house.

Conflict of interest The authors declare that they have no conflict of interest.

Open Access This article is distributed under the terms of the Creative Commons Attribution Noncommercial License which permits any noncommercial use, distribution, and reproduction in any medium, provided the original author(s) and source are credited.

\section{References}

Aoyama CH, WuY CM, Sessa G, Ruberti I, Morelli G, Chua NH, Dong T (1995) Ectopic expression of the Arabidopsis transcriptional activator Athb-I alters leaf cell fate in tobacco. Plant Cell 7:1773-1785

Bowman JL, Smyth DR (1999) CRABS CLAW, a gene that regulates carpel and nectary development in Arabidopsis, encodes a novel protein with zinc finger and helix-loop-helix domains. Development $126: 2387-2396$ 
Brandt S, Kehr J, Walz C, Imlau A, Willmitzer L, Fisahn J (1999) A rapid method for detection of plant gene transcripts from single epidermal, mesophyll and companion cells of intact leaves. Plant J 20:245-250

Brandt S, Kloska S, Altmann T, Kehr J (2002) Using array hybridization to monitor gene expression at the single cell level. J Exp Bot 53:2315-2323

Clough SJ, Bent AF (1998) Floral dip: a simplified method for Agrobacterium-mediated transformation of Arabidopsis thaliana. Plant J 16:735-743

Czechowski T, Bari RP, Stitt M, Scheible WR, Udvardi MK (2004) Real-time RT-PCR profiling of over 1400 Arabidopsis transcription factors: unprecedented sensitivity levels reveal novel rootand shoot-specific genes. Plant J 38:366-379

Doyle JJ, Doyle JL (1987) A rapid DNA isolation procedure for small quantities of fresh leaf tissue. Phytochem Bull 19:11-15

Ebert B, Melle C, Lieckfeldt E, Zöller D, von Eggeling F, Fisahn J (2008) Protein profiling of single epidermal cell types from Arabidopsis thaliana using surface-enhanced laser desorption and ionization technology. J Plant Physiol 165:1227-1237

Esch JJ, Chen M, Sanders M, Hillestad M, Ndkium S, Idelkope B, Neizer J, Marks MD (2003) A contradictory GLABRA3 allele helps define gene interactions controlling trichome development in Arabidopsis. Development 130:5885-5894

Fricke W, Leigh RA, Tomos AD (1994) Concentrations of inorganic and organic solutes in extracts from individual epidermal, mesophyll and bundle-sheath cells of barley leaves. Planta 192:310-316

Gallagher JA, Koroleva OA, Tomos DA, Farrar JF, Pollock CJ (2001) Single cell analysis technique for comparison of specific mRNA abundance in plant cells. J Plant Physiol 158:1089-1092

Glover BJ (2000) Differentiation in plant epidermal cells. J Exp Bot 51:497-505

Henriksson E, Olsson ASB, Johannesson H, Johansson H, Hanson J, Engström P, Söderman E (2005) Homeodomain leucine zipper class I genes in Arabidopsis. Expression patterns and phylogenetic relationships. Plant Physiol 139:509-518

Horak CE, Snyder M (2002) Global analysis of gene expression in yeast. Func Int Genomics 2:171-180

Hüsken D, Steudle E, Zimmermann U (1978) Pressure probe technique for measuring water relations of cells in higher plants. Plant Physiol 61:158-163

Jang S, Hur J, Kim SJ, Han MJ, Kim SR, An G (2004) Ectopic expression of OsYAB1 causes extra stamens and carpels in rice. Plant Mol Biol 56:133-143

Jefferson RA, Kavanagh TA, Bevan MW (1987) GUS fusions: beta glucuronidase as a sensitive and versatile gene fusion marker in higher plants. Embo J 6:3901-3907

Kirik V, Lee MM, Wester K, Herrmann U, Zheng Z, Oppenheimer D, Schiefelbein J, Hülskamp M (2005) Funtional diversification of MYB23 and GL1 genes in trichome morphogenesis and initiation. Development 132:1477-1485
Koroleva OA, Tomos AD, Farrar JF, Gallagher J, Pollock CJ (2001) Carbon allocation and sugar status in individual cells of barley leaves affects expression of sucrose: fructosyltransferase gene. Annals Appl Biol 138:27-32

Kryvych S, Nikiforova V, Herzog M, Perazza D, Fisahn J (2008) Gene expression profiling of the different stages of Arabidopsis thaliana trichome development on the single cell level. Plant Physiol Biochem 46:160-173

Laval V, Koroleva OA, Murphy E, Lu C, Milner JJ, Hooks MA, Tomos AD (2002) Distribution of actin gene isoforms in the Arabidopsis leaf measured in microsamples from intact individual cells. Planta 215:287-292

Lieckfeldt E, Simon-Rosin U, Kose F, Zoeller D, Schliep M, Fisahn J (2008) Gene expression profiling of single epidermal, basal and trichome cells of Arabidopsis thaliana. J Plant Physiol 165:1530-1544

Nicot N, Hausman JF, Hoffmann L, Evers D (2005) Housekeeping gene selection for real-time RT-PCR normalization in potato during biotic and abiotic stress. J Exp Bot 56:2907-2914

Payne CT, Zhang F, Lloyd AM (2000) GL3 encodes a bHLH protein that regulates trichome development in Arabidopsis through interaction with GL1 and TTG1. Genetics 156:1346-1362

Sawa S, Ito T, Shimura Y, Okada K (1999) FILAMENTOUS FLOWER controls the formation and development of Arabidopsis inflorescences and floral meristems. Plant Cell 11:69-86

Schellman S, Schnittger A, Kirik V, Wada T, Okada K, Beermann A, Thumfahrt J, Juergens G, Huelskamp M (2002) TRIPTYCHON and CAPRICE mediate lateral inhibition during trichome and root hair patterning in Arabidopsis. EMBO J 21:5036-5046

Siegfried KR, Eshed Y, Baum SF, Otsuga D, Drews GN, Bowman JL (1999) Members of the YABBY gene family specify abaxial cell fate in Arabidopsis. Development 126:4117-4128

Stracke R, Werber M, Weisshaar B (2001) The R2R3-MYB gene family in Arabidopsis thaliana. Curr Opin Plant Biol 4:447-456

Szymanski DB, Lloyd AM, Marks MD (2000) Progress in the molecular genetic analysis of trichome initiation and morphogenesis in Arabidopsis. Trends Plant Sci Rev 5:214-219

Tominaga R, Iwata M, Okada K, Wada T (2007) Functional analysis of the epidermal-specific MYB genes CAPRICE and WEREWOLF in Arabidopsis. Plant Cell 19:2264-2277

Toriba T, Harada K, Takamura A, Nakamura H, Ichikawa H, Suzaki T, Hirano HY (2007) Molecular characterization of the YABBY gene family in Oryza sativa and expression analysis of OsYABBY1. Mol Genetics Genomics 277:457-468

Tomos AD, Leigh RA (1999) The pressure probe: a versatile tool in plant cell physiology. Annu Rev Plant Physiol Plant Mol Biol 50:447-472

Wada T (2002) Role of a positive regulator of root hair development, CAPRICE, in Arabidopsis root epidermal cell differentiation. Development 129:5409-5419

Wada T, Tachibana T, Shimura Y, Okada K (1997) Epidermal cell differentiation in Arabidopsis determined by a MYB homolog, CPC. Science 277:1113-1116 